\title{
Author Index Vol. 35, 1995
}

Aebischer, P. 65 Aguglia, U. 177 Aguirrebengoa, K. 239 AlDeeb, S.M. 217 d’Allens, H. 264 Angelini, C. 230 Angibaud, G. 43 Antonello, R. 193 Apostolski, S. 104 Appollonio, I. 344 Autret, A. 174 Azulay, J.P. 181

Bahou, Y. 217 Bardos, P. 174 Barre, J. 143 Bartolomei, F. 181 Baumann, P, 206 Belleville, S. 363 Bertin, L. 264 Bianchetti, A. 50 Biary, N. 217 Binkofski, F. 20 Black, P.McL. 124 Blizzard, R.A. 341 Blomstrand, C. 259 Bogousslavsky, J. 1, 27, 336 Bono, F. 177 Bornstein, N.M. 46 Bosco, A. 193 Boureau, F. 264 Bradvik, B. 80 Brian:, J.F. 131 Brown, M.T, 122 Bujdakova, J. 212 Biittner, Th. 226 Byth, K. 321

Caillaud, C. 175

Camus, C. 236

Caplan, L.R. 118, 126, 293, 309

Capon, A. 254

Cargnelutti, D. 193

Carraro, N. 193

Cazzato, G. 193

Ceccaldi, M. 363

Chang, W.-N. 55

Chazot, G. 264

Chen, C.-M. 281

Chen, S.-T. 349, 359

Chen, T.-C. 359

Cheng, S.-Y. 349

Cheng,Y.-F. 55

Chierichetti, F. 50 Choi, I.S. 137 Choi, Y.C. 137 Chu, N.-S. 199 Clanet, M. 43 Colson, C. 254 Cook, MJ. 327 Correra, G. 246 Coyle, P.K. 113

Defer, G.-L. 143 Degos, J.-D. 143 Delacretaz, F. 336 Demeurisse, G. 254 DeStefano, N. 246 Dethy, S. 38 Dotti, M.T. 246 Drory, V.E. 240 Duncan, J.S. 172

Einstein, R. 321 Elie, B. 175 Emile, J. 264 Espadaler, J.M. 287 Evyapan, D. 244

Fabrizi, G.M. 182 Federico, A. 246 Ferrarese, C. 344 Ferruzza, E. 230 Fiaschi, A. 93 Fiori, L. 354 Fish, D.R. 327 Fisher, M. 3 Folgera, T. 168 Frattola, L. 344 Free, S.L. 327 French Sumatriptan Freund, H.-J. 20 Frisoni, G.B. 50 Fukui, T. 86 Fukutake, T. 69 Furutama, D. 270

Gallassi, R. 179 Gasecki, A.P. 127 Ghika, J. 27 Guazzi, G.C. 182

Habib, M. 131 Halligan, P.W. 341 Hanyu, S. 173 Harper, P. 237

Hashimoto, R. 58 Hashimoto, T. 220 Hayashi, A. 63 Hedera, P. 212 Henderson, J.M. 321 Hennerici, M. 249 Heuts-van Raak, L. 276 Heyen, P. 162 Hildebrand, J. 118 Hirayama, K. 69 Holm, J. 259 Houtte, P.van 38 Howard, R.S. 172 Huang, C.-C. 199, 281 Hublet, C. 254 Hughes, R.A.C. 237 Hupperts, R. 276

Ichikawa, H. 86 Ikeguchi, K. 173 Imai, H. 173 Imai, N. 173 Isensee, Ch. 183 Ishii, A. 63,156

Jackson, D.M. 321 Jouannic, I. 236

Kanazawa, I. 243

Kato, A.C. 65

Kauranen, K. 206

Kim, S.K. 137

Kinoshita, M. 99

Kjallman, L. 259

Klastersky, J. 38

Knecht, H. 336

Korczyn, A.D. 46

$264 \quad$ Kuhn, W. 226

Kumral, E. 244 Kunesch, E. 20 Kuntzer, T. 336 Kwieciiliski, H. 127

Larner, A.J. 172 Lavrnic, D. 104 Lawrence, C. 113 Lazzarino, L.G. 241 LedudaLP. 143 Lee, S.M. 237 Lee, S.S. 137 Leroy, J.-P. 236 LeTulzo, Y. 236 Levrier, O. 131 Levy, D. 341 Liou, C.-W. 199 Lipton, R.B. 113 Lodder, J. 276 Loeb, C. 187 Louboutin, J.P. 175 Lowy, F.D. 113 Lugaresi, E. 179 Lundh, T. 259 Luthert, P. 237 Lyu, R.-K. 349 ,359

Maetzu, J.C.I, de 239 Makino, S. 270 Malandrini, A. 182 Manford, M.R.A. 327 Manganotti, P. 93 Manneschi, L. 182 Marinkovic, Z. 104 Martinello, F. 230 Masur, H. 162 Mazoyer, B.M. 131 Meglio, M. 33 Meier, C. 336 Meincke, U. 183 Melle, G. van 27 Menage, P. 174 Mesiano, T. 193 Messina, C. 177 Miletic, V. 104 Miralles, R. 287 Miyasaka, M. 220 Mizusawa, H. 63 Mochizuki, I. 108 Montejo, M. 239 Monti, F. 193 Moreau, M.S. 43 Morreale, A. 179 Morris, J.G.M. 321 MulLM. 183 Murayama, N. 131 Myllyla, V. 206

Nagai, C. 243 Nakajima, H. 270 Nakamura, K. 243 Navarro,|X. 287 Nicolai, A. 241 Nikolic, J. 104 Nisipeanu, P. 240 Nogues, B. 175

Oberwittler, C. 162 Ogawa, T. 99 Oguni, E. 63 Ohkoshi, N. 156

Ohsawa, N. 270 0ian, P. 168 Okada, S. 99 (Mate, J. 239 
Paesmans, M. 38 Pang, C.-Y. 199 Parenti, G. 354 Parrotta, E. 182 Paternot, J. 254 Perego, M. 344 Piccart, M.J. 38 Piolti, R. 344 Pizzolato, G. 50 Polo, A. 93 Poncet, M. 363 Pozzi, C. 344 Prieta, R. de la 239 Przuntek, H. 226 Puca, A. 33

Quattrone, A. 177

Rakocevic, V. 104 RascoLO. 43 Raybaud, Ch. 131 Regli, F. 27, 336 Reider-Groswasser, I. 46 Reuther, G. 162 Righetti, C. 93 Rosen, I. 80 Rosi, R. 182 Rovati, L. 344 Royere, M.-L. 363 Rubies-Prat, J. 287 Ryding, E. 80

Sabbah, P. 131 Sadamoto, K. 99 Sadiq, S.A. 104 Saito, E. 99 Salamon, G. 131 Sato, Y. 86 Saudeau, D. 174 Scarpini, C. 182 Schabet, M. 120 Seguin, P. 236 Serratrice, G. 181 Shih, K.-D. 199 Shinoda, K. 270 Shoji, S. 63,156 Shorvon, S.D. 327 Simond, G. 131 Sjaastad,0. 149 Smith, S.J.M. 172 Sonesson, B. 80

Steck, A.J. 336 Steinmetz, H. 20 Stevens, J.M. 327 Stone, S.P. 341 Stovner, LJ. 149 Sugita, K. 86

Takeuchi, T. 86 Tamburrini, G. 33 Tanaka, Y. 58 Tancredi, A. 33 Tang, L.-M. 349,359 Tassinari, C.A. 179 Terao, Y. 243 Thomas, R. 236 Tillement, J.-P. 143 Tinazzi, M. 93 ToffoLB.de 174 Torbergsen, T. 168 Torre, P. 193 Tosi, L. 93 Trabaud, V. 131 Trabucchi, M. 50 Traubner, P. 212 Trevisan, C.P. 230 Trikic, R. 104 Tsukada, N. 108 Tsukagoshi, H. 86

Ugawa, Y. 243

Valentino, P. 177 Van Broeckhoven, C.L. 8 Vanharanta, H. 206 Vari, R. 33 Vecchione, V. 246 Vidakovic, A. 104 Vingerhoets, F. 336 Vita, G. 177 Volkmann, R. 259

Wai, Y.-Y. 199 Watier, H. 174 Wei, Y.-H. 199 Wendt, G. 183 Wirguin, I. 104

Yanagisawa, N. 108,220 Yoshida, M. 58,173

Zanette, G. 93 Zanetti,0. 50 Zappia, M. 177 Zarate, P.G. de 239

AuthorlndexVol. 35,1995 